\title{
PROPOSTA DE MODELO CINÉTICO PARA REAÇÕES DE ADIÇÃO QUE ORIGINAM OS POLÍMEROS SUPERABSORVENTES
}

\author{
R. Santos ${ }^{1}$, C. Neves ${ }^{1}$, G. Costa $^{1}$, K. Pontes ${ }^{1}$ \\ ${ }^{1}$ Programa de Pós-Graduação em Engenharia Industrial, Universidade Federal da Bahia \\ E-mail para contato: vergne@ufba.br
}

\begin{abstract}
RESUMO - Polímero superabsorvente (Superabsorbent Polymer - SAP) pode ser produzido a partir da copolimerização de um monômero monovinílico com um agente de crosslink. A copolimerização de SAP forma uma rede polimérica capaz de absorver e reter grande quantidade de água e solução aquosa. Devido a essa característica, o polímero se tornou bastante útil na produção de artigos de higiene pessoal como fraldas descartáveis e, apesar da sua importância no mercado mundial, há escassez na literatura acerca dos modelos cinéticos para a sua produção. Um dos poucos trabalhos voltados para modelagem de polímero superabsorvente faz uso de complexas funções geratrizes que torna difícil a sua aplicação. Existe ainda na literatura um modelo alternativo baseado no método dos momentos aplicável a SAP, no entanto, este se fundamenta em dezenas de reações que tornam o modelo pouco usual. Este artigo tem como objetivo propor um modelo cinético simplificado, porém consistente, baseado no método dos momentos para a copolimerização do ácido acrílico com um agente de crosslink difuncional.
\end{abstract}

\section{INTRODUÇÃO}

Polímero superabsorvente ou SAP (Superabsorbent Polymer) é um gel cuja principal característica é a absorção e retenção de grande quantidade de água ou solução aquosa. SAPs foram introduzidos no Japão no final da década de 1970 para produção de artigos de higiene pessoal (Zohuriaan-Mehr et al., 2010). Devido à implantação de novas plantas em países emergentes como Brasil e China (BASF, 2014) a previsão da produção mundial de SAPs para 2015 está estimada em cerca de 1,9 milhão de toneladas, segundo o site da PRWeb. O monômero mais utilizado para produção de SAP é o ácido acrílico (Buchholz e Graham, 1998), sua polimerização é via adição com um agente de crosslink responsável por unir as cadeias em uma grande rede polimérica tridimensional.

O objetivo do presente trabalho é discutir e comparar os modelos cinéticos já publicados visando propor um modelo simplificado e consistente para a polimerização do ácido acrílico com agente de crosslink difuncional. Neste intuito são consideradas pertinentes algumas simplificações na cinética dos modelos propostos na literatura que não comprometem a descrição do processo como um todo. Outro fato relevante é o detalhamento e consolidação da simbologia para a descrição das espécies e etapas do processo, até então bastante confusa na literatura, o que dificulta a sua compreensão e consequente aplicação. 


\section{REVISÃO DA LITERATURA}

De acordo com uma vasta revisão da literatura, apenas os trabalhos de Gonçalves et al. (2011) e Mikos et al. (1986, 1987 a, 1987 b) abordam uma modelagem cinética voltada para polimerização de SAP. O primeiro utiliza equações de balanço populacional, um modelo estatístico que, apesar de conhecido na literatura, é de difícil aplicação devido à complexidade das funções geratrizes em que se baseiam. O segundo, baseado no consagrado método dos momentos, propõe um modelo para a copolimerização de um monômero monovinílico com um agente de crosslink divinílico e torna possível o cálculo do peso molecular numérico médio das cadeias.

Em um primeiro trabalho, Mikos e Peppas (1987 a) propuseram um modelo cinético para predição das características estruturais do copolímero formado a partir do monômero HEMA (2hidroxietil metacrilato) e do agente de crosslink EGDMA (dimetacrilato de etilenoglicol). Os autores consideram que os monômeros possuem a mesma reatividade e que a reatividade das duplas ligações do EGDNA não se altera quando a segunda dupla ligação reage, ao contrário do demonstrado por Kwant (1979). Em outro trabalho, Mikos et al. (1986) passam a diferenciar a reatividade entre os diferentes monômeros. Em um terceiro trabalho, Mikos et al. (1987 b) propuseram um modelo cinético mais abrangente para a reação de estireno com m-divinilbenzeno ou p-divinilbenzeno. Neste modelo os autores consideram diferentes reatividades e dependência cinética, além de fazer uso de cinquenta e cinco reações, o que torna a resolução matemática do problema complexa. Diferentemente de Gonçalves et al. (2011), este modelo considera cadeias independentes que são conectadas por agentes de crosslink.

\section{MECANISMO CINÉTICO}

Este artigo propõe um modelo cinético simplificado para a copolimerização do ácido acrílico com o agente de crosslink difuncional EGDMA na presença do termoiniciador persulfato de sódio, tomando como base o método apresentado por Mikos et al. (1987 b). As reações consideradas são iniciação, propagação e terminação. $\mathrm{Na}$ produção de SAPs a limitação do peso molecular é indesejada porque, quanto maior for a cadeia, maior a probabilidade de ela possuir agentes de crosslink e menor a probabilidade desta cadeia não estar unida à rede. Dessa forma, não é usual utilizar um agente de transferência de cadeia, de forma que a reação de transferência de cadeia não é considerada no mecanismo aqui proposto. Será utilizada a notação $P_{p, q, r}, Q_{p, q, r}$ e $R_{p, q, r}$ para polímeros vivos com seus respectivos grupos terminais monômero monovinílico (AA), dupla ligação pendente (DPL) e agente de crosslink (AC), e os subíndices p, q e r contabilizam respectivamente as unidades de repetição monômero monovinílico, dupla ligação pendente e agente de crosslink. A Figura 1 ilustra os polímeros vivos de grupos terminais AA, DPL e AC e, assim como as demais figuras, foi criada a partir do software ChemSketch.

\subsection{Iniciação}

A iniciação se dá pela termodecomposição do iniciador (I) em dois radicais (A), que atacam as espécies reacionais, conforme lista a Tabela 1. A Figura 2a exemplifica o ataque de um radical livre A à dupla ligação pendente do polímero $Q_{p, q, r}$. Outras reações de iniciação com radicais vinílico ocorrem de forma análoga. $\mathrm{O}$ ataque do iniciador pode ocorrer também na dupla ligação do grupo terminal do $Q_{p, q, r}$, como ilustra a Figura $2 \mathrm{~b}$. Neste caso a cadeia passa a ter grupo terminal AC, logo denotada por $R_{p, q+1, r+1}$. Conclui-se então que, para a iniciação de um polímero 
vivo $Q_{p, q, r}$, há dois produtos possíveis: $Q_{p, q-1, r+1}$ e $R_{p, q+1, r+1}$. Em ambos os casos, considera-se que há a formação de uma nova cadeia $R_{0,0,1}$ pois surge um novo sítio ativo na antiga dupla ligação pendente.

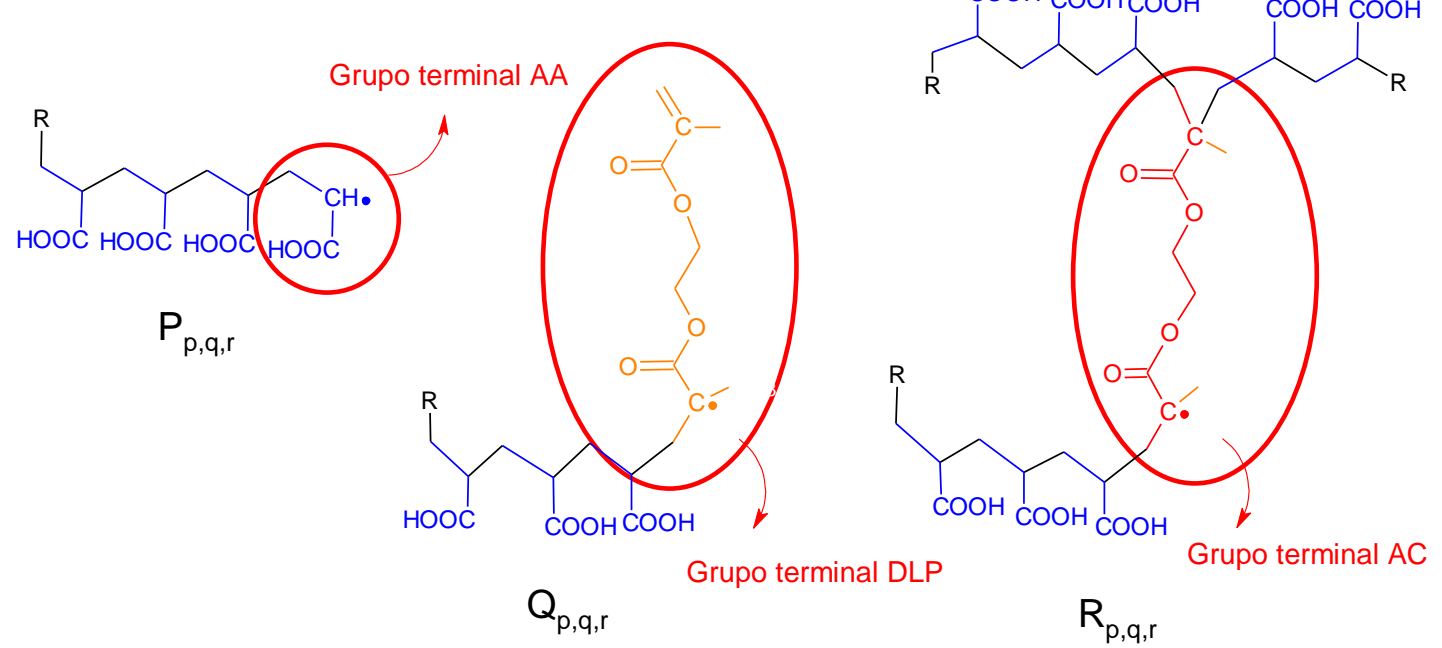

Figura 1 - Polímeros vivos $\boldsymbol{P}_{\boldsymbol{p}, \boldsymbol{q}, \boldsymbol{r}}, \boldsymbol{Q}_{\boldsymbol{p}, \boldsymbol{q}, \boldsymbol{r}}$ e $\boldsymbol{R}_{\boldsymbol{p}, \boldsymbol{q}, \boldsymbol{r}}$ com grupos terminais AA, DPL e AC.

Tabela 1 - Reações para copolimerização do ácido acrílico com agente de crosslink EGDMA

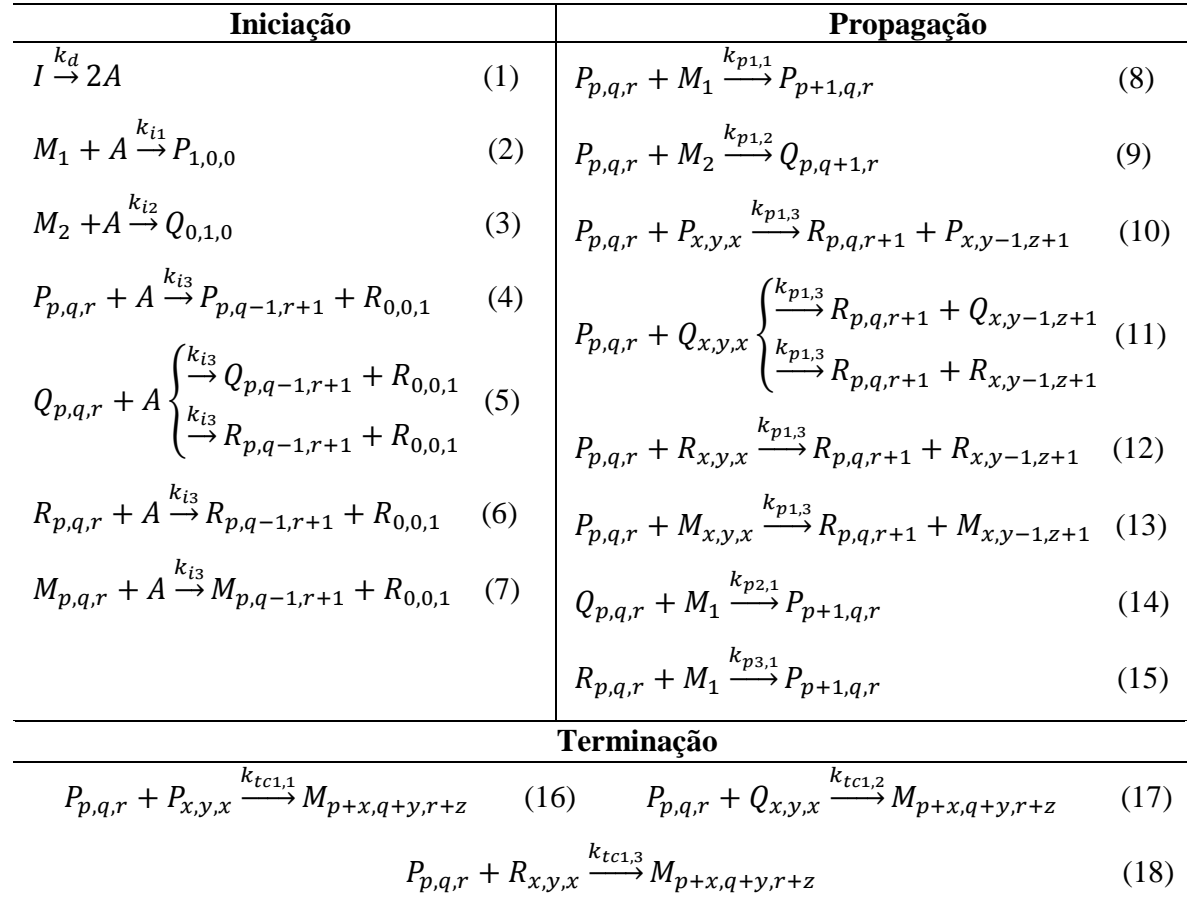

\subsection{Propagação}

Propagação é a etapa de crescimento da cadeia. A Figura 3 ilustra a propagação de um polímero vivo $P_{p, q, r}$ a outro polímero $P_{x, y, z}$. As demais reações de propagação são listadas na 
Tabela 1. Assim como na iniciação, o polímero atacado, $P_{x, y, z}$, terá menos uma ligação dupla pendente e mais uma unidade agente de crosslink, $P_{x, y-1, z+1}$, e o polímero que atacou, $P_{p, q, r}$, terá mais uma unidade agente de crosslink. Sua notação passará a ser $R_{p, q, r+1}$ pois o grupo terminal deixou de ser AA e passou a ser AC.
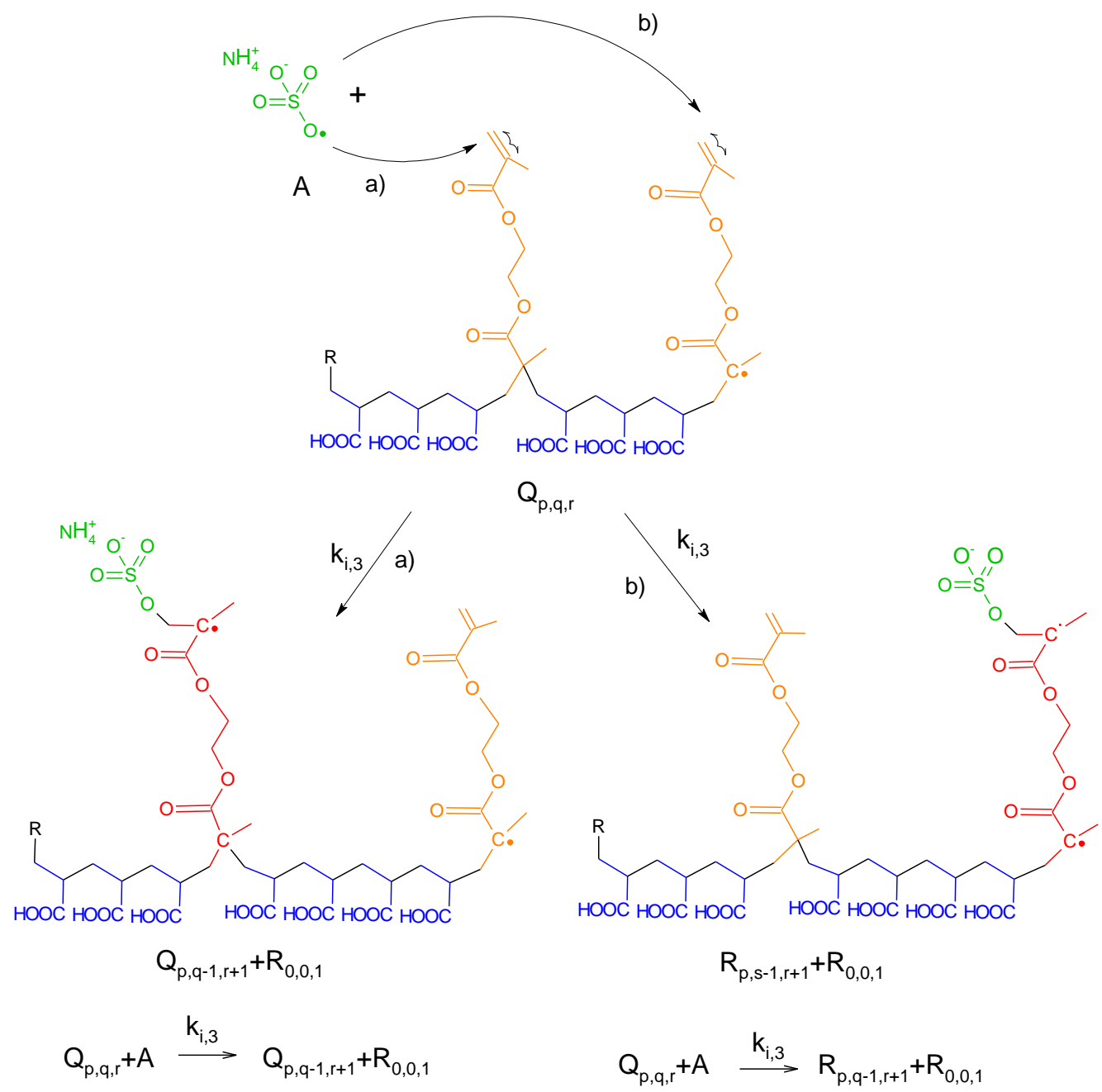

Figura 2 - Reações de iniciação de um polímero $\boldsymbol{Q}_{\boldsymbol{p}, \boldsymbol{q}, \boldsymbol{r}}$ a) em uma dupla ligação pendente ao longo da cadeia e b) na dupla ligação pendente do grupo terminal.

\subsection{Terminação}

A terminação por combinação é prioritária em acrilatos, conforme Gonçalves et al. (2011), logo esta será a única terminação considerada no modelo aqui proposto. A Figura 4 mostra a terminação por combinação de polímeros vivos com terminações AA cujo produto é o polímero morto $M_{p+x, q+y, r+z}$. Não serão consideradas as terminações que envolvam a aproximação de grupos terminais DPL e AC devido à baixa concentração de agente de crosslink. 


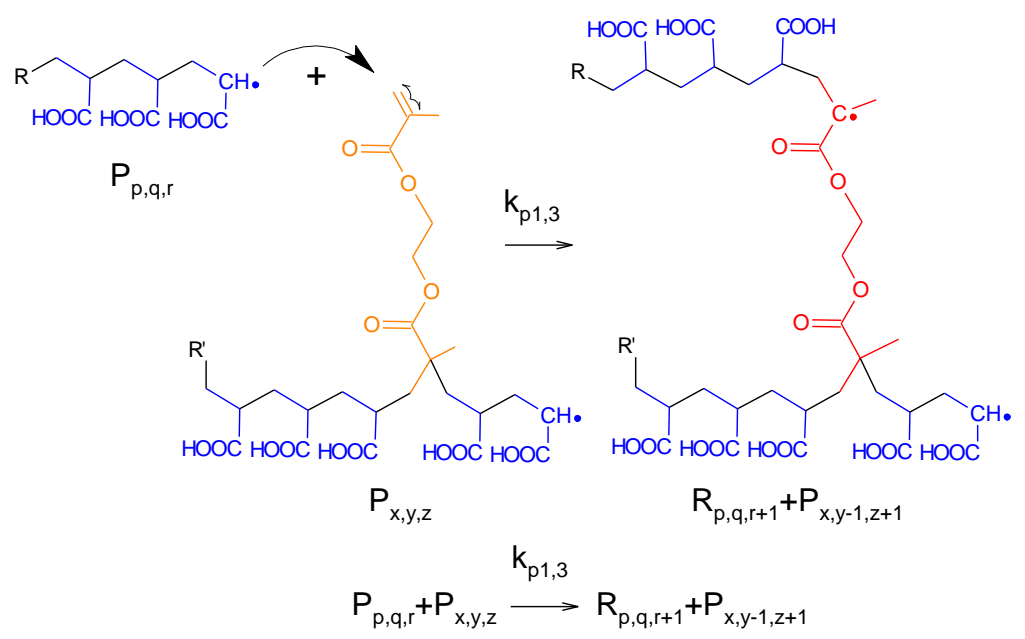

Figura 3 - Reação de propagação de um polímero vivo $\boldsymbol{P}_{\boldsymbol{p}, \boldsymbol{q}, \boldsymbol{r}}$ a outro polímero vivo $\boldsymbol{P}_{\boldsymbol{x}, \boldsymbol{y}, \boldsymbol{z}}$.

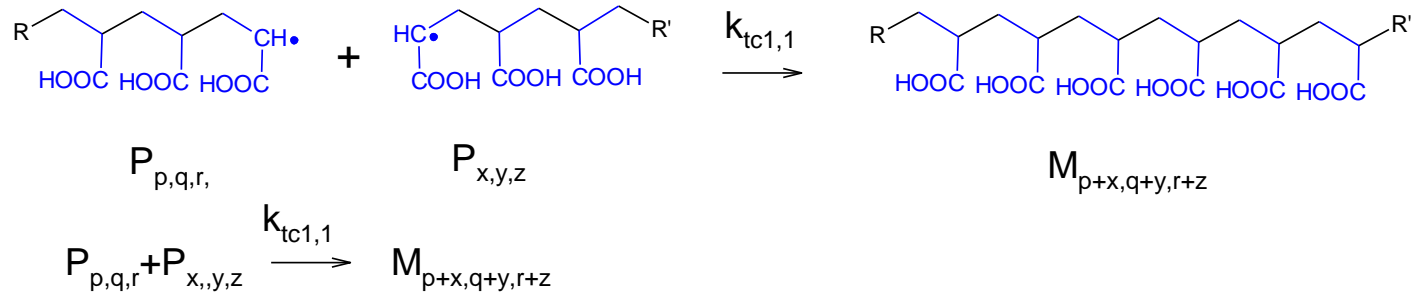

Figura 4 - Terminação por combinação de polímeros vivos $P_{p, q, r}$ e $P_{x, y, z}$.

\subsection{Modelo Cinético}

O mecanismo cinético proposto é descrito por dezoito reações conforme resumido na Tabela 1. As concentrações totais de polímeros vivos $P, Q, R$ e polímero morto, $M$, são definidos por:

$$
X=\sum_{p=0}^{\infty} \sum_{q=0}^{\infty} \sum_{r=0}^{\infty} X_{p, q, r}
$$

onde $X$ assume os valore de $P, Q, R$ e $M$ e os termos em negrito representam a concentração de cada espécie.

Assumindo um reator batelada em solução, o balanço de massa que descreve o sistema é dado na Tabela 2 onde $f$ na Equação 3 é a eficiência do iniciador. $\mathrm{O}$ termo $\left(k_{i 2} A+k_{p 1,2} P\right) M_{2}$ da Equação 5, por exemplo, é multiplicado por dois porque há duas duplas ligações que podem ser atacadas no $M_{2}$. De forma análoga, os termos $P, Q, R$ e $M$ na Equação 3 têm $q$ duplas ligações pendentes que podem ser atacadas. Já o polímero $P_{p, q+1, r-1}$ na Equação 6, por exemplo, é multiplicado por $q+1$ porque há $q+1$ duplas ligações pendentes que permitem a formação de $P_{p, q, r}$. Por outro lado na Equação 8, por exemplo, o polímero $Q_{p, q+1, r-1}$ não é multiplicado por que há uma forma de $Q_{p, q+1, r-1}$ gerar $P_{p, q, r}$ conforme destacado na Figura 2b. Por definição os momentos da distribuição de peso molecular são dados por:

$$
\psi_{i, j, k}=\sum_{p=0}^{\infty} \sum_{q=0}^{\infty} \sum_{r=0}^{\infty} p^{i} q^{j} r^{k}\left(P_{p, q, r}+Q_{p, q, r}+R_{p, q, r}+M_{p, q, r}\right)
$$


onde $i, j$ e $k$ são as ordens do momento. A partir dos momentos é possível calcular o peso molecular numérico médio, $\bar{M}_{n}$, segundo Mikos et al. (1987 b):

$$
\bar{M}_{n}=\frac{w_{1} \psi_{1,0,0}+w_{2}\left(\psi_{0,1,0}+0,5 \psi_{0,0,1}\right)}{\psi_{0,0,0}}
$$

onde $w_{1}$ e $w_{2}$ são os pesos moleculares do monômero e do agente de crosslink, respectivamente. Como o agente de crosslink é compartilhado entre duas cadeias, multiplica-se o fator 0,5 a $\Psi_{0,0,1}$. É desejável que a cadeia dos SAPs tenha uma boa dispersão entre os agentes de crosslink para que sua absorção seja maximizada. Tendo isso em vista, torna-se importante calcular o peso molecular médio entre agentes de crosslinks, $\bar{M}_{c}$, segundo Mikos et al. (1987 b):

$$
\bar{M}_{c}=\frac{\bar{M}_{n}}{1+\frac{\psi_{0,0,1}}{\psi_{0,0,0}}}
$$

Tabela 2 - Taxa das concentrações das espécies

$$
\begin{aligned}
& \frac{d I}{d t}=-k_{d} I \\
& \frac{d A}{d t}=2 f k_{d} I-\left[\begin{array}{c}
k_{i 1} M_{1}+2 k_{i 2} M_{2}+ \\
k_{i 3} q(P+Q+R+M)
\end{array}\right] A \\
& \frac{d M_{1}}{d t}=-\left(k_{i 1} A+k_{p 1,1} P+k_{p 2,1} Q+k_{p 3,1} R\right) M_{1} \\
& \frac{d M_{2}}{d t}=-2\left(k_{i 2} A+k_{p 1,2} P\right) M_{2} \\
& \frac{d P_{p, q, r}}{d t}=\left(k_{p 1,1} P_{p-1, q, r}+k_{p 2,1} Q_{p-1, q, r}+k_{p 3,1} R_{p-1, q, r}\right) M_{1}-\frac{d P}{d t}=\left(k_{i 1} A+k_{p 2,1} Q+k_{p 3,1} R\right) M_{1}- \\
& -\left(k_{i 3} A+k_{p 1,3} P\right)\left[q P_{p, q, r}-(q+1) P_{p, q+1, r-1}\right]- \\
& -\left[\begin{array}{c}
k_{p 1,1} M_{1}+2 k_{p 1,2} M_{2}+k_{p 1,3}(Q+R+M) \\
+k_{t c 1,1} P+k_{t c 1,2} Q+k_{t c 1,3} R
\end{array}\right] P_{p, q, r} \\
& -\left[\begin{array}{c}
2 k_{p 1,2} M_{2}+k_{p 1,3} q(P+Q+R+M) \\
+k_{t c 1,1} P+k_{t c 1,2} Q+k_{t c 1,3} R
\end{array}\right] P \\
& \text { (3) }+\frac{1}{2} k_{t c 1,1} \sum_{p=0}^{\infty} \sum_{q=0}^{\infty} \sum_{r=0}^{\infty} P_{x, y, z} P_{p-x, q-y, r-z}+ \\
& \text { (4) }+\boldsymbol{k}_{t c 1,2} \sum_{p=0}^{\infty} \sum_{q=0}^{\infty} \sum_{r=0}^{\infty} P_{x, y, z} Q_{p-x, q-y, r-z}+ \\
& \text { (5) } \quad+\boldsymbol{k}_{t c 1,3} \sum_{p=0}^{\infty} \sum_{q=0}^{\infty} \sum_{r=0}^{\infty} \boldsymbol{P}_{x, y, z} \boldsymbol{R}_{p-x, q-y, r-z} \\
& \text { (6) } \frac{d Q}{d t}=2\left(k_{i 2} A+k_{p 1,2} P\right) M_{2}- \\
& \frac{d Q_{p, q, r}}{d t}=2\left(k_{p 1,2} P_{p, q-1, r}\right) M_{2}- \\
& -\left(k_{i 3} A+k_{p 1,3} P\right) q\left(Q_{p, q, r}-Q_{p, q+1, r-1}\right)- \\
& -\left(k_{p 2,1} M_{1}+k_{t c 1,2} P\right) Q_{p, q, r} \\
& \frac{d R_{p, q, r}}{d t}=\left(k_{p 1,3} P_{p, q, r-1}\right) q(P+Q+R+M)- \\
& -\left(k_{i 3} A+k_{p 1,3} P+k_{p 2,1} M_{1}+k_{t c 1,2} P\right) Q \\
& \frac{d R}{d t}=\left(k_{i 3} A+k_{p 1,3} P\right) q(P+Q+R+M)+ \\
& \text { (7) }+\left(k_{i 3} A+k_{p 1,3} P\right) Q-\left(k_{p 3,1} M_{1}+k_{t c 1,3} P\right) R \\
& \frac{d M}{d t}=\frac{1}{2} k_{t c 1,1} P^{2}+k_{t c 1,2} P Q++k_{t c 1,3} P R \\
& -\left(k_{i 3} A+k_{p 1,3} P\right)\left[\begin{array}{c}
q R_{p, q, r}-(q+1) P_{p, q+1, r-1} \\
-Q_{p, q+1, r-1}
\end{array}\right]- \\
& -\left(k_{p 3,1} M_{1}+k_{t c 1,3} P\right) R_{p, q, r}
\end{aligned}
$$

As taxas dos momentos de ordem zero e um são obtidas a partir da Equação 14 e da Tabela 2, de acordo com as seguintes equações diferenciais e a razão entre elas na Equação 16 é chamado de número médio de crosslink efetivo:

$$
\frac{d \psi_{0,0,0}}{d t}=\left(k_{i 1} \boldsymbol{M}_{\mathbf{1}}+2 k_{i 2} \boldsymbol{M}_{\mathbf{2}}+k_{i 3} \psi_{0,1,0}\right) A-\frac{1}{2} k_{t c 1,1} \boldsymbol{P}^{2}-k_{t c 1,2} \boldsymbol{P Q}-k_{t c 1,3} \boldsymbol{P R}
$$




$$
\begin{aligned}
& \frac{d \psi_{1,0,0}}{d t}=\left(k_{i 1} \boldsymbol{A}+k_{p 1,1} \boldsymbol{P}+k_{p 2,1} \boldsymbol{Q}+k_{p 3,1} \boldsymbol{R}\right) \boldsymbol{M}_{\mathbf{1}} \\
& \frac{d \psi_{0,1,0}}{d t}=2\left(k_{i 2} \boldsymbol{A}+k_{p 1,2} \boldsymbol{P}\right) \boldsymbol{M}_{2}-\left(k_{i 3} \boldsymbol{A}+k_{p 1,3} \boldsymbol{P}\right) \psi_{0,1,0} \\
& \frac{d \psi_{0,0,1}}{d t}=2\left(k_{i 3} \boldsymbol{A}+k_{p 1,3} \boldsymbol{P}\right) \Psi_{0,1,0}
\end{aligned}
$$

\section{RESULTADOS}

Assumindo-se a QSSA (Quasi-Steady State Assumption - Hipótese do Estado QuasiEstacionário), o sistema composto por oito equações diferencias (Equação 2-5 e 17-20) foi resolvido em Matlab. Devido à escassez de modelos cinéticos voltados para SAPs, não foram encontrada na literatura constantes para polimerização do ácido acrílico com EGDMA. Dessa maneira, este modelo foi validado com as constantes disponibilizadas por Hild e Okasha (1985) para copolimerização do estireno com m-divinilbenzeno. O gráfico da conversão é mostrado na Figura 5, onde o subíndice 1 é referente ao estireno e 2 ao m-divinilbenzeno.

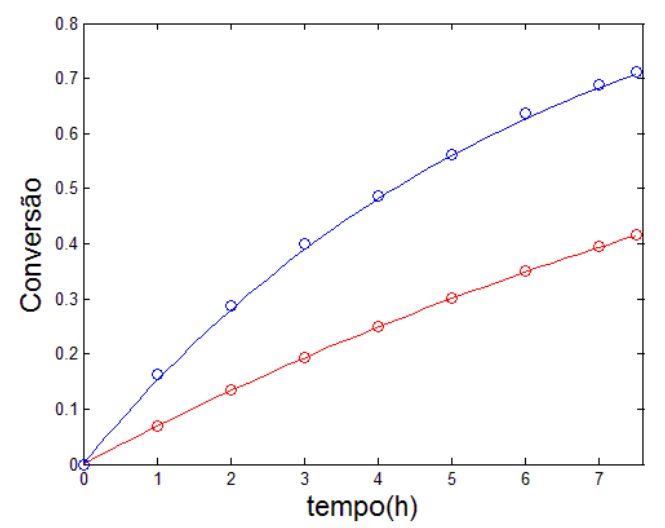

Figura 5 - Conversão da reação entre ácido acrílico e EGDMA

Após validado do modelo, avaliou-se o comportamento do peso molecular numérico médio e entre agentes de crosslink, variando, respectivamente, a concentração de iniciador e agente de crosslink. Os resultados são mostrados na Figura 6. Nota-se que, à medida que a concentração de iniciador diminui, para uma mesma concentração de monômero, o peso molecular aumenta devido ao menor número de sítios para crescimento da cadeia. Tal característica é importante para a produção de SAPs com maior poder de absorção, pois cria cadeias com maior probabilidade de pertencer à rede e com maior quantidade de monômeros hidrofílicos.

\section{CONCLUSÃO}

Este artigo propôs um modelo cinético simplificado em relação aos poucos disponíveis na literatura, porém consistente, para a polimerização do ácido acrílico com agente de crosslink difuncional. A validação com dados de literatura indicaram a boa qualidade de ajuste do modelo que pode ser ainda estendido para copolimerização de mais monômeros com agentes de crosslinks multifuncional. Outra contribuição deste trabalho é o detalhamento e consolidação da simbologia 
para a descrição das espécies e etapas da reação, até então bastante confusa na literatura.


Figura 6 - Distribuição do peso molecular médio (esquerda) e número médio de crosslink efetivo (direita) para diferentes concentrações de iniciador e agente de crosslink

\section{REFERÊNCIAS}

BASF. Disponível em www.basf.com.br/sac/web/brazil/pt_BR/imprensa/releases/20111014R01. Acesso 27 de Abril de 2014;

Buchholz, Fredric L. e Grahan, Andrew T. Modern Superabsorbent Polymer Technology, Editora Wiley-VCH, 1998;

ChemSketch. Software gratuíto disponível em http://chemsketch.softonic.com.br/;

Hild, G, Okasha R. Kinetic investigation of the Free Radical Crosslinking Copolymerization in the Pre-Gel state, 1. Makromol. Chem., v. 186, p. 93-110, 1985;

Kwant, P.W. Kinetics of the Copolymerization of Styrene with Small Quantities of Divinylbenzenes. J. Polym. Sci., Polym. Chem. Edn., v. 17, p. 1331-1338, 1979;

Miguel A. D. Gonçalves,Virgínia D. Pinto, Rolando C. S. Dias, Mário Rui P. F. N. Costa. Modeling Studies on the Synthesis of Superabsorbent Hydrogels Using Population Balance Equations. Macromol. Symp., v. 306-307, p. 107-125, 2011;

Mikos, A. G., Peppas, N.A.J. A Model for Prediction of the Structural Characteristics of EGDMA-Crosslinked PHEMA Microparticles Produced by Suspension Copolymerization/Crosslink. Controlled Release. v. 5, p. 53-62, 1987;

Mikos, A.G.; Takoudis, C.G. Peppas, N.A. Evidence of Unequal Vinyl Group Reactivity in Copolymerization/Crosslinking Reactions of Mono- and Divinyl Comonomers. Polymer. v. 28, p. 998-1004, 1987;

Mikos, A.G.; Takoudis, C.G. Peppas, N.A. Kinetic Modeling of Copolymerization/CrossLinking Reactions. Macromolecules. v. 19, p. 2174-2182, 1986;

PRWeb. Disponível em www.prweb.com. Acesso em 1 de Junho de 2014.

Zohuriaan-Mehr, M. J, H Omidian, S Doroudiani, K Kabiri. Advances in Non-Hygienic Applications of Superabsorbent Hydrogel Materials. J Mater Sci, v. 45, p. 5711-5735, 2010. 